\title{
System metric for holographic memory systems
}

\author{
Fai H. Mok \\ Holoplex, Suite 102, 600 South Lake Avenue, Pasadena, California 91106 \\ Geoffrey W. Burr* and Demetri Psaltis \\ Department of Electrical Engineering, California Institute of Technology, Pasadena, California 91125
}

Received December 19, 1995

\begin{abstract}
We introduce $\mathrm{M} / \#$ as a metric for characterizing holographic memory systems. $\mathrm{M} / \#$ is the constant of proportionality between diffraction efficiency and the number of holograms squared. Although $\mathrm{M} / \#$ is a function of many variables in a holographic recording system, it can be measured from the recording and erasure of a single hologram. We verify experimentally that the diffraction efficiency of multiple holograms follows the prediction of M/\# measured from a single hologram. (C) 1996 Optical Society of America
\end{abstract}

Photorefractive crystals are widely used for volume holographic storage because incident light modulates their indices of refraction. ${ }^{1}$ Multiple holograms of equal diffraction efficiency can be stored by use of a recording schedule. ${ }^{2}$ If only a few holograms are stored, coupling theory can be used to solve for the dependence of diffraction efficiency on the number of holograms $M .^{3}$ For large $M$, diffraction efficiency is proportional to $1 / M^{2}$. The exact value of diffraction efficiency depends on many material and system parameters, giving many opportunities for optimization. However, it is inconvenient to record thousands of holograms to observe the effect of each individual element in the system configuration.

In this Letter we introduce $\mathrm{M} / \#-$ a parameter that succinctly characterizes the dynamic range performance of a holographic memory system. M/\# can be regarded as a result of the derivation of the recording schedule, which we summarize briefly. When multiple holograms are to be recorded, we calculate the proper set of exposure times by assuming that each hologram evolves during recording as $A_{0}[1-$ $\left.\exp \left(-t / \tau_{r}\right)\right] \approx\left(A_{0} / \tau_{r}\right) t$ and decays during erasure as $\exp \left(-t / \tau_{e}\right)$. Here we have assumed that each exposure time is much shorter than $\tau_{r}$ and that the intensities involved are not large enough to induce nonlinear effects. For a large number of holograms $M$, the final equalized diffraction efficiency is ${ }^{2}$

$$
\eta=\left[\left(\frac{A_{0}}{\tau_{r}}\right) \frac{\tau_{e}}{M}\right]^{2}=\left(\frac{\mathrm{M} / \#}{M}\right)^{2} .
$$

$A_{0}$ is the saturation grating strength, $\tau_{r}$ is the recording time constant, $\tau_{e}$ is the erasure time constant, and we define $\mathrm{M} / \#$ as

$$
\mathrm{M} / \#=\left(\frac{A_{0}}{\tau_{r}}\right) \tau_{e} .
$$

Often, $A_{0}$ and $\tau_{r}$ are difficult to measure individually. For instance, a complex time constant or two-beam coupling may cause the diffraction efficiency to deviate from exponential behavior as the hologram evolves. However, the ratio $\left(A_{0} / \tau_{r}\right)$, which is essentially the recording slope during the first $<1 \%$ of $\tau_{r}$, is straightforward to measure.

0146-9592/96/120896-03\$10.00/0
Specifically, $\left(A_{0} / \tau_{r}\right)$ is the slope of $\sqrt{\eta}$ as a function of time during the initial stages of hologram formation. It can be obtained from a before-and-after diffraction efficiency measurement of a single short exposure. The beam used for single hologram measurement should be the same one used for readout in the multiple hologram system being characterized.

The erasure time $\tau_{e}$ is the decay time during illumination with both signal and reference beams. To avoid coupling, neither beam should Bragg match the hologram being measured. However, the incidence angles should be similar to those used for recording so the local intensity throughout the crystal will be the same. The erasure time $\tau_{e}$ of the grating is then twice the inverse of the slope of $\log \eta$ as a function of time, and $\mathrm{M} / \#$ is the product of $\tau_{e}$ and $A_{0} / \tau_{r}$.

An improvement in $\mathrm{M} / \#$ is a direct improvement in system performance, whether it is applied toward more holograms or higher diffraction efficiency. M/\# is not limited to values less than 1 . Nonphysical predictions of $>100 \%$ diffraction efficiency do not occur because our derivation of $\mathrm{M} / \#$ assumes that $M$ is large. $\mathrm{M} / \#$ depends on the physical characteristics of the photorefractive crystal, such as impurity doping level, oxidation state, absorption coefficient, electrooptic coefficient, photoconductivity, and the presence of a photovoltaic effect. The holographic recording process also contributes to $\mathrm{M} / \#$ through modulation depth, coherence length, grating period, externally applied fields, the size and shape of the interaction volume, and the stability of the interference pattern during recording. On readout, changes in readout wavelength or failure to return to the exact Braggmatching condition can affect the measured diffraction efficiency. Despite the number of variables involved, though, the overall dynamic range performance can be summarized by $\mathrm{M} / \#$. As the product of hologram recording slope, $A_{0} / \tau_{r}$, and the erasure time $\tau_{e}, \mathrm{M} / \#$ can be measured from the growth and decay of a single hologram.

$\mathrm{M}$ /\# measured from the recording and erasure of a single hologram can be used to predict, with good accuracy, the diffraction efficiency of $M$ holograms by use of an ideal recording schedule. This ideal 
recording schedule has a final exposure of length $\tau_{e} / M$ but an infinitely long initial exposure. Any practical initial exposure implies that the final exposure is some fraction $f$ of $\tau_{e} / M$. After a recording schedule with such a final exposure is used, all $M$ holograms will be equalized in diffraction efficiency to $\eta=$ $f^{2}(\mathrm{M} / \# / M)^{2}$. An equivalent way of thinking of this is that an ideal exposure schedule for $M+X$ holograms was formulated but that the first $X$ holograms were skipped and recording started with exposure $X+1$. $X$, the number of skipped holograms, and $f$, the fraction of $\mathrm{M} / \#$ that is used, are related by $f=M /(M+$ $X)$. The relationship between $X$ and $f$ is plotted in Fig. 1 for $M=1000$ holograms.

The parameter $f$ (or, equivalently, $X$ ) is useful because it permits control over the average recording time (for a given intensity). Proper choice of the parameter $f$ can reduce the total recording time to practical values with a moderate sacrifice of diffraction efficiency. Indeed, $f$ can be considered the fraction of $\mathrm{M} / \#$ used. Figure 2 shows the relationship between the average recording time and $f$ for storage of 1000 holograms. Note that using the last few percent of dynamic range is very costly in terms of recording time.

Conventionally, the exposure schedule is calculated by a forward recursion algorithm: an initial time is chosen, and recording times are calculated up to the $M$ th exposure. Knowledge of both the recording and the erasure time constants is required. Because the recording time constant cannot be measured reliably, the only way to find it is by trial and error. Instead, we can use a simpler backward-recursion algorithm, which permits explicit selection of the fraction $f$ and does not require knowledge of the recording time constant. We summarize the algorithm as follows: given the measured erasure time constant $\tau_{e}$ and the desired number of holograms $M$, the last exposure is chosen to be $t_{M}=f \tau_{e} / M$. We calculate previous exposure times recursively, using the expression

$$
t_{m-1}=t_{m} \exp \left(t_{m} / \tau_{e}\right),
$$

until the first recording time $t_{1}$. This initial exposure, and the whole recording sequence, will be finite if $f<1$.

We perform experimental measurements to compare $\mathrm{M} / \#$ from a single hologram measurement with the diffraction efficiency of multiple holograms. We used a $2 \mathrm{~cm} \times 1.5 \mathrm{~cm} \times 1 \mathrm{~cm} \mathrm{LiNbO}_{3}$ crystal with $0.01 \%$ Fe doping from Deltronics Crystals. We recorded holograms in the $90^{\circ}$ geometry, using equal irradiance plane-wave reference and signal beams at $488 \mathrm{~nm}$. A small rectangular portion of a large volume grating was sampled by a weak plane-wave readout beam. M/\# was measured from the recording and erasure of single holograms. The average and the standard deviation of $\mathrm{M} / \#$ are shown in the first row of Table 1 . The erasure time constant was measured during exposure with both the reference and the signal beams, with the crystal and reference beams rotated to avoid coupling with the original grating during erasure.

We used the same experimental setup to store multiple holograms. The erasure time constant of $540 \mathrm{~s}$ was taken from the results of single hologram measurement. We derived recording schedules for $100,200,400$, and 1000 holograms, using $f=0.8$ and $f=0.6$. After a set of multiple holograms was recorded, we measured the diffraction efficiency by sampling a subset of 20 well-distributed holograms. Figure 3 is a $\log -\log$ plot of the average diffraction efficiency as a function of the number of holograms. As expected, the data follow a $1 / M^{2}$ relationship. We compute $\mathrm{M} / \#$ by dividing the intercepts of the fitted $1 / M^{2}$ curves by $f^{2}$; the result is shown in Table 1. The results show that $\mathrm{M} / \#$ measured from a single hologram is a good indicator of the diffraction efficiency of multiple holograms. The observed trend of a lower $\mathrm{M} / \#$ for a higher value of $f$ stems from two second-order effects. High values of $f$ lead to schedules in which both the initial exposures and the

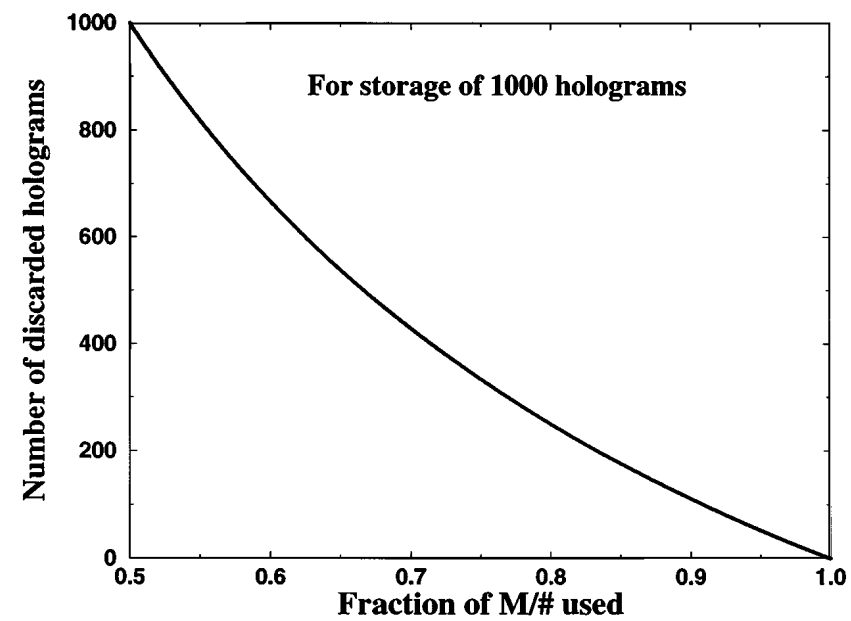

Fig. 1. Number of holograms discarded from recording schedule versus fraction $f$ of $\mathrm{M} / \#$ used, for storage of 1000 holograms.

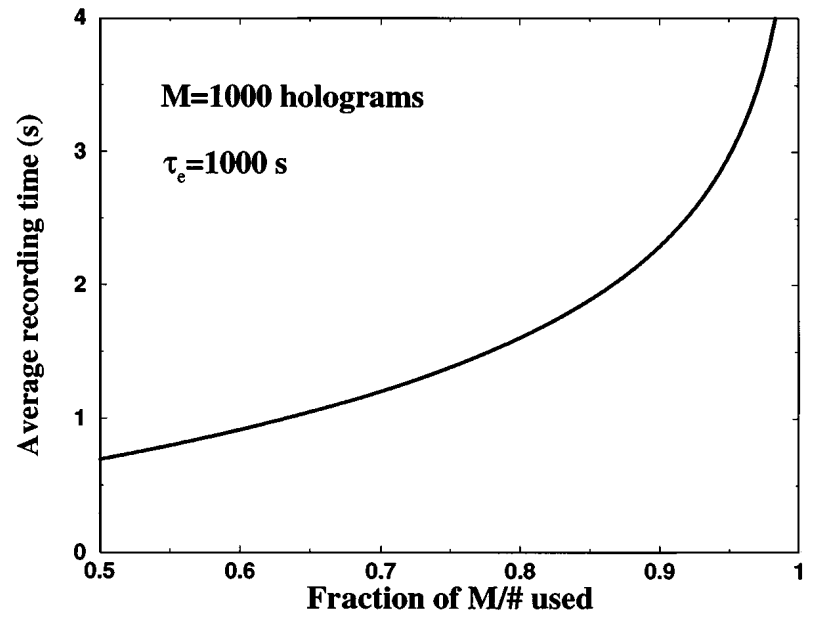

Fig. 2. Average recording time versus fraction $f$ of $\mathrm{M} / \#$ used.

Table 1. Experimentally Measured M/\#

\begin{tabular}{lc}
\hline \multicolumn{1}{c}{ Experiment } & $\mathrm{M} / \#$ \\
\hline Single hologram recording/erasure & $1.368 \pm 0.061$ \\
Multiple holograms, $f=0.8$ & 1.256 \\
Multiple holograms, $f=0.6$ & 1.492 \\
\hline
\end{tabular}




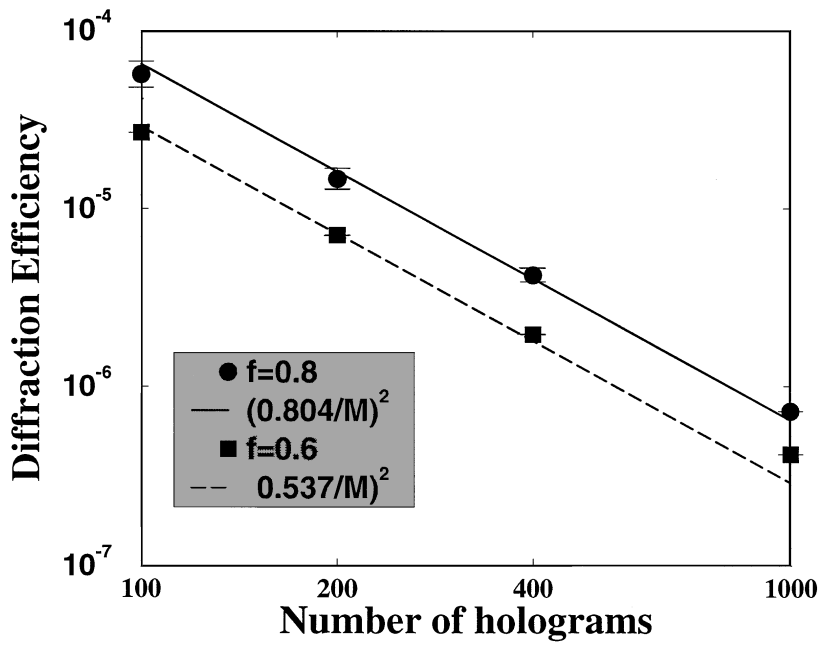

Fig. 3. Diffraction efficiency versus number of holograms stored, for $f=0.8$ and $f=0.6$. The final recording time for $M$ holograms is $t_{\text {last }}=f \tau_{e} / M$, where $\tau_{e}=540 \mathrm{~s}$.

total exposure time are long. The approximation of linear recording may not be valid during these long exposure times, leading to weaker initial holograms. The long total exposure time leads to an increase in the bulk electric field induced by the photovoltaic effect. This field tends to cause a slight Bragg mismatch for earlier holograms and to reduce $A_{0} / \tau_{r}$ for later-written holograms. The sum effect is a reduction in the average measured diffraction efficiency for schedules constructed with large values of $f$.

In conclusion, we have introduced a system metric that concisely characterizes the dynamic range performance of a holographic storage system. By defining $\mathrm{M} / \#$ in terms of recording slope and erasure time, we can determine it from the recording and erasure of a single hologram. We have introduced a new recursion algorithm for the recording schedule that does not require knowledge of the recording behavior. This algorithm also gives explicit control over the trade-off between average recording time and diffraction efficiency. Finally, we have experimentally verified that $\mathrm{M} / \#$, measured from the recording and erasure of a single hologram, is an accurate predictor of the diffraction efficiency of multiple holograms.

*Present address, IBM Almaden Research Center, 650 Harry Road, San Jose, California 95120.

\section{References}

1. D. L. Staebler, J. J. Amodei, and W. Phillips, presented at the VII International Quantum Electronics Conference, Montreal, Canada, May 1972.

2. D. Psaltis, D. Brady, and K. Wagner, Appl. Opt. 27, 1752 (1988).

3. E. Maniloff and K. Johnson, J. Appl. Phys. 70, 4702 (1991). 